\title{
Pegylated liposomal doxorubicin for platinum-resistant or refractory Müllerian carcinoma (epithelial ovarian carcinoma, primary carcinoma of Fallopian tube and peritoneal carcinoma): A single-institutional experience
}

\author{
TAKESHI FUKUDA, TOSHIYUKI SUMI, MASATOMO TERAMAE, YUSUKE NAKANO, MASANARI MORISHITA, \\ HIROYUKI TERADA, HIROYUKI YOSHIDA, YOSHINARI MATSUMOTO, TOMOYO YASUI and OSAMU ISHIKO
}

\author{
Department of Obstetrics and Gynecology, Osaka City University Graduate School of Medicine, \\ Abeno-ku, Osaka 545-8585, Japan
}

Received April 3, 2012; Accepted September 21, 2012

DOI: $10.3892 / \mathrm{ol} .2012 .971$

\begin{abstract}
The aim of the present study was to evaluate the efficacy and safety of pegylated liposomal doxorubicin (PLD) in patients with Müllerian carcinoma treated at our hospital. Nineteen patients with platinum-resistant Müllerian carcinoma were treated with intravenous PLD $50 \mathrm{mg} / \mathrm{m}^{2}$ every 4 weeks. Tumor response was assessed by MRI following every 2-3 cycles of treatment. The severity of adverse events was assessed according to the Common Terminology Criteria for Adverse Events (v3.0). The best overall responses in the 19 patients were identified as 5 partial responses (PR), 6 stable diseases (SD) and 8 progressive diseases (PD). Response rate was $26.3 \%$. The proportion of patients with CR, PR or SD was $57.9 \%$. The median time to progression was 188.0 days. The median survival time was 381.0 days. Toxicity grades were identified as one grade III hand-foot syndrome, two grade III neutropenia, one grade IV hand-foot syndrome, one grade IV stomatitis and one grade IV neutropenia. The present study confirmed that PLD is an effective drug when administered as a salvage therapy for the treatment of Müllerian carcinoma and is associated with a reduced toxicity profile compared with current therapeutic options.
\end{abstract}

\section{Introduction}

Epithelial ovarian carcinoma is one of the most common gynecological malignancies and the fifth most frequent cause of cancer mortality in females (1). Epithelial ovarian and peritoneal

Correspondence to: Dr Toshiyuki Sumi, Department of Obstetrics and Gynecology, Osaka City University Graduate School of Medicine, 143 Asahimachi, Abeno-ku, Osaka 545-8585, Japan E-mail: sumi-toshi@med.osaka-cu.ac.jp

Key words: pegylated liposomal doxorubicin, Müllerian carcinoma, ovarian carcinoma, platinum resistance carcinomas and primary carcinoma of the Fallopian tube are all classified as Müllerian carcinomas. These diseases exhibit a number of similarities and are often treated using similar therapeutic protocols. Advanced epithelial ovarian cancer is highly chemosensitive and $70-80 \%$ of patients initially respond to platinum-based chemotherapy. However, in $60-80 \%$ of cases these tumors increase in severity and treatment with second-line agents becomes necessary $(2,3)$. Tumors which do not progress in severity until more than 6 months following completion of first-line platinum-based treatment are considered likely to remain platinum-sensitive and generally receive a chemotherapy regimen containing a platinum agent. However, tumors associated with severe progression in less than 6 months following completion of the first-line chemotherapy are considered to be platinum-resistant (4). Secondary treatments currently induce a response in $14-40 \%$ of patients and these responses are generally of short duration (5). This results in rapid disease recurrence following discontinuation of therapy. At present, it is considered extremely difficult to cure recurrent ovarian cancer and existing therapies are considered suitable for increasing the duration of survival and palliative therapy only. Consequently, suitable therapeutic agents for the treatment of this disease are required to demonstrate efficacy and low toxicity.

Doxorubicin has a wide spectrum of activities in human tumors and is considered to be suitable as an active agent for the treatment of advanced ovarian cancer (6-8). However, use of this anthracycline agent is limited by side-effects, including gastrointestinal toxicity, myelosuppression and cumulative cardiac damage (9). Pegylated liposomal doxorubicin (PLD) is a unique formulation of conventional doxorubicin that avoids phagocytosis. This results in prolonged circulation time with drug retention in liposomes and selective accumulation in the tumor vascular bed following extravasation through the leaky tumor vasculature $(10,11)$. PLD was designed specifically to enhance the efficacy of doxorubicin and reduce its toxicities, including myelosuppression, alopecia and cardiotoxicity.

The aim of the present study was to evaluate the effects of PLD in a non-trial setting to evaluate the efficacy and safety of the treatment. 


\section{Materials and methods}

Patients. The present study was retrospective and aimed to evaluate the efficacy and safety of PLD in Japanese patients with ovarian carcinoma previously treated with platinum-based chemotherapy at our hospital. The primary endpoint of the study was determination of best overall response. The secondary endpoint included analysis of adverse events and drug reactions and duration of response. The study included 18 patients with epithelial ovarian carcinoma and 1 with peritoneal carcinoma and was approved by the Ethics Committee of Osaka City University. All patients were identified as platinum-resistant. Written informed consent was provided by all patients prior to treatment with PLD at our hospital between July 2009 and March 2011.

Treatment and clinical course analysis. Patients were treated with PLD at a dose of $50 \mathrm{mg} / \mathrm{m}^{2}$ every 4 weeks, which was delivered in $250 \mathrm{ml}$ of $5 \%$ dextrose solution and administered as an intravenous infusion over $1 \mathrm{~h}$. All patients received standard premedication of intravenous dexamethasone $(10 \mathrm{mg})$ and ramosetron hydrochloride $(0.3 \mathrm{mg})$. Prior to each PLD cycle, toxicity evaluations were performed. Tumor response was reassessed by MRI following every 2-3 cycles of treatment in all cases. Tumor response evaluation was performed according to the RECIST guidelines. The severity of adverse events was assessed according to the Common Terminology Criteria for Adverse Events (v3.0). Subsequent courses of chemotherapy were planned every 28 days pending an absolute neutrophil count (ANC) of 1,500 cells/ $\mathrm{ml}$ and resolution of $\geq$ grade II nonhematological toxicity. In the event of persistent neutropenia (ANC $<1,500$ cells $/ \mathrm{ml}$ ) or $\geq$ grade II nonhematological toxicity, the therapy was delayed and the patients were reevaluated weekly using these criteria for reinstitution of therapy. PLD dose reductions of $10 \mathrm{mg} /$ $\mathrm{m}^{2}$ were instituted in the event of grade III or IV nonhematological toxicity.

Statistical analysis. Probabilities of survival and progression-free survival were analyzed using the Kaplan-Meier method (12). StatView 5.0 (Abacus Concepts, Berkley, CA, USA) was used for statistical analysis. $\mathrm{P}<0.05$ was considered to indicate a statistically significant difference.

\section{Results}

Patient characteristics. The demographics and baseline characteristics of the patients are presented in Table I. Between July 2009 and March 2011, 19 patients were treated with PLD. The median age of the patients was 54.8 years (range, 39-75 years). Among the 19 patients, 18 had epithelial ovarian carcinoma and 1 had peritoneal carcinoma. Histologically, 11 patients had serous adenocarcinoma, 3 had clear cell adenocarcinoma, 2 had mucinous adenocarcinoma, 2 had poorly differentiated adenocarcinoma and 1 had endometrioid adenocarcinoma. The most common FIGO stage was III. Prior to treatment with PLD, 2 patients received one chemotherapeutic regimen, 11 patients received two regimens, 4 patients received three regimens and 2 patients received four regimens.
Table I. Patient characteristics $(n=19)$.

\begin{tabular}{lc}
\hline Characteristics & Total \\
\hline Age, years & \\
Median & 54.8 \\
Range & $39-75$ \\
Primary cancer, n (\%) & \\
Epithelial ovarian carcinoma & $18(94.7)$ \\
Peritoneal carcinoma & $1(5.3)$ \\
Tumor histology, n (\%) & \\
Serous & $11(57.9)$ \\
Mucinous & $2(10.5)$ \\
Clear cell & $3(15.8)$ \\
Endometrial & $1(5.3)$ \\
Poorly differentiated adenocarcinoma & $2(10.5)$ \\
Initial FIGO stage, n (\%) & \\
I & $2(10.5)$ \\
II & $2(10.5)$ \\
III & $14(73.7)$ \\
IV & $1(5.3)$
\end{tabular}

Number of prior chemotherapy cycles, n (\%)

$\begin{array}{rr}1 & 2(10.5) \\ 2 & 11(57.9) \\ 3 & 4(21.1) \\ 4 & 2(10.5)\end{array}$

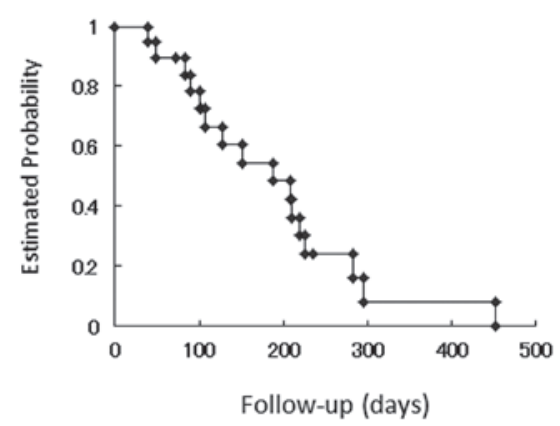

Figure 1. Kaplan-Meier estimates of time to progression.

Tumor characteristics. The antitumor effects (best overall responses) and response rates are presented in Table II. The best overall responses in the 19 patients were 5 partial responses (PR), 6 stable diseases (SD) and 8 progressive diseases (PD). The response rate was $26.3 \%$. The proportion of patients with complete response (CR), PR or SD was $57.9 \%$ (11/19 patients).

The Kaplan-Meier curve for time to progression is demonstrated in Fig. 1. Median time to progression was 188.0 days. The Kaplan-Meier curve for overall survival is shown in Fig. 2. Median survival time was 381.0 days.

Table III provides a summary of the grade III and IV toxicities. The toxicities were grade III hand-foot syndrome (HFS) in 1 patient $(5.3 \%)$, grade IV HFS in $1(5.3 \%)$, grade IV stomatitis in $1(5.3 \%)$, grade III neutropenia in $2(10.5 \%)$ and 
Table II. Response rates.

\begin{tabular}{ll}
\hline Response & $\mathrm{n}(\%)$ \\
\hline Best overall response & \\
CR & $0(0)$ \\
PR & $5(26.3)$ \\
SD & $6(31.6)$ \\
PD & $8(42.1)$ \\
Response rate & $5(26.3)$ \\
\hline
\end{tabular}

$\mathrm{n}=19$. CR, complete response; PR, partial response; SD, stable disease; $\mathrm{PD}$, progressive disease.

Table III. Grade III and IV adverse drug reactions.

\begin{tabular}{llc}
\hline Disease & $\begin{array}{c}\text { Grade III } \\
\mathrm{n}(\%)\end{array}$ & $\begin{array}{c}\text { Grade IV } \\
\mathrm{n}(\%)\end{array}$ \\
\hline Hand-foot syndrome & $1(5.3)$ & $1(5.3)$ \\
Stomatitis & $0(0)$ & $1(5.3)$ \\
Neutropenia & $2(10.5)$ & $1(5.3)$ \\
\hline
\end{tabular}

$\mathrm{n}=6$, percentages calculated from $\mathrm{n}=19$.

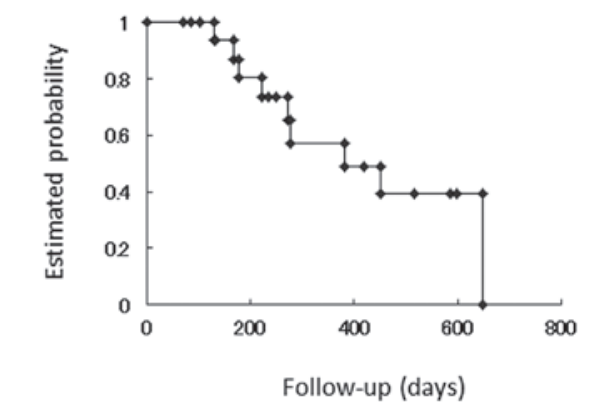

Figure 2. Kaplan-Meier estimates of overall survival.

grade IV neutropenia in $1(5.3 \%)$. One case of grade IV stomatitis required a delay of therapy and a dose reduction.

\section{Discussion}

PLD is an active drug in patients with persistent or recurrent platinum-pretreated epithelial ovarian carcinoma. The clinical applications of PLD have expanded considerably in recent years and the drug has been introduced into the management of the majority of protocols for epithelial ovarian carcinoma treatment alone and in combination with non-platinum and platinum agents (13). The recent CALYPSO trial demonstrated the efficacy of PLD/carboplatin combination treatments for recurrent platinum-sensitive disease. This combination is currently associated with a more favorable risk-benefit profile than paclitaxel/carboplatin in patients with partially platinumsensitive recurrent ovarian carcinoma (14). A previous study in elderly patients revealed that PLD demonstrated an improved therapeutic index with less toxicity than paclitaxel/carboplatin (15).

In the present study, the response rate was $26.3 \%$. Clinical studies conducted in the United States and Europe identified response rates to PLD of $28.4 \%$ in the platinum-sensitive group and 6.5-18.3\% in the platinum-resistant group (16-18). A phase II clinical trial in Japan reported response rates of 27.3 and $21.0 \%$ in the platinum-sensitive and platinum-resistant groups, respectively (19). These response rates are consistent with those obtained in the present study.

PLD is an attractive second-line agent due to the absence of treatment-associated mortality reports and low rates of febrile neutropenia even in heavily pretreated patients $(16,20,21)$. The common toxicities identified in the present study were hematological toxicities, HFS and stomatitis. Grade III-IV HFS was observed in 2 patients $(10.5 \%)$, grade IV stomatitis was observed in $1(5.3 \%)$ and grade III-IV neutropenia was observed in $3(15.8 \%)$. A previous phase II clinical trial in Japan identified that $16.2,8.1$ and $67.6 \%$ of patients experienced grade III-IV HFS, stomatitis and neutropenia, respectively (19). Only 1 case of grade IV stomatitis required therapy delay and a dose reduction. However, all patients were able to receive PLD continually. Side-effects previously associated with doxorubicin, including allergic reactions and cardiotoxicity, were not identified, indicating that PLD is a well-tolerated agent.

In conclusion, recurrent ovarian cancer is extremely difficult to cure, with current therapies functioning only to lengthen the duration of survival and palliative therapy. At present, the main aim of recurrent epithelial ovarian carcinoma management is to reduce harm. Additional aims of therapy must include the alleviation of cancer-related symptoms and extension of symptom-free, progression-free and overall survival where possible. Suitable agents must not only be effective but should also be associated with low toxicities. The present study confirms that PLD is suitable for use in salvage therapy of Müllerian carcinoma and has a reduced toxicity profile compared with current therapeutic options.

\section{References}

1. Jemal A, Siegel R, Ward E, Hao Y, Xu J and Thun MJ: Cancer statistics, 2009. CA Cancer J Clin 59: 225-249, 2009.

2. Seewaldt VL, Greer BE, Cain JM, et al: Paclitaxel (Taxol) treatment for refractory ovarian cancer: phase II clinical trial. Am J Obstet Gynecol 170: 1666-1670, 1994.

3. Neijt IP, ten Bokkel Huiniuk WW, van der Berg ME, et al: Long-term survival in ovarian cancer. Mature data from the Netherlands Joint Study Group for Ovarian Cancer. Eur J Cancer 27: 1367-1372, 1991.

4. Dear RF, Gao B and Harnett P: Recurrent ovarian cancer: Treatment with pegylated liposomal doxorubicin; a Westmead Cancer Care Centre Experience. Asia Pac J Clin Oncol 6: 66-73, 2010.

5. Safra T, Groshen S, Jeffers S, Tsao-Wei DD, Zhou L, Muderspach L, Roman L, Morrow CP, Burnett A and Muggia FM: Treatment of patients with ovarian carcinoma with pegylated liposomal doxorubicin: analysis of toxicities and predictors of outcome. Cancer 91: 90-100, 2001.

6. No authors listed: Cyclophosphamide plus cisplatin versus cyclophosphamide, doxorubicin and cisplatin chemotherapy of ovarian carcinoma: a meta-analysis. The Ovarian Cancer Meta Analysis Project. J Clin Oncol 9: 1668-1674, 1991.

7. A'Hern RP and Gore ME: Impact of doxorubicin on survival in advanced ovarian cancer. J Clin Oncol 13: 726-732, 1995.

8. Fanning J, Bennett TZ and Hilgers RD: Meta-analysis of cisplatin, doxorubicin and cyclophosphamide versus cisplatin and cyclophosphamide chemotherapy of ovarian carcinoma. Obstet Gynecol 80: 954-960, 1992. 
9. Singal PK and Iliskovic N: Doxorubicin-induced cardiomyopathy. N Engl J Med 339: 900-905, 1998.

10. GabizonAandMartinF:Polyethyleneglycol-coated(pegylated) liposomal doxorubicin: rationale for use in solid tumors. Drugs 54 (Suppl 4): 15-21, 1997.

11. Collins Y and Lele S: Long-term pegylated liposomal doxorubicin use in recurrent ovarian carcinoma. J Natl Med Assoc 97: 1414-1416, 2005

12. Kaplan EL and Meier P: Nonparametric estimation from incomplete observations. J Am Stat Assoc 53: 457, 1958.

13. Ferrandina G, Corrado G, Licameli A, Lorusso D, Fuoco G, Pisconti S and Scambia G: Pegylated liposomal doxorubicin in the management of ovarian cancer. Ther Clin Risk Manag 6: 463-483, 2010

14. Gladieff L, Ferrero A, De Rauglaudre G, et al: Carboplatin and pegylated liposomal doxorubicin versus carboplatin and paclitaxel in partially platinum-sensitive ovarian cancer patients: results from a subset analysis of the CALYPSO phase III trial. Ann Oncol 5: 1185-1189, 2011.

15. Kurtz JE, Kaminsky MC, Floquet A, et al: Ovarian cancer in elderly patients: carboplatin and pegylated liposomal doxorubicin versus carboplatin and paclitaxel in late relapse: a Gynecologic Cancer Intergroup (GCGI) CALYPSO sub-study. Ann Oncol 22: 2417-2423, 2011

16. Gordon AN, Granai CO, Rose PG, et al: Phase II study of stealth liposomal doxorubicin in platinum- and paclitaxel-refractory epithelial ovarian cancer. J Clin Oncol 18: 3093-3100, 2000.
17. Johnston SRD and Gore ME: Caelyx: phase II studies in ovarian cancer. Eur J Cancer 37 (Suppl 9): S8-S14, 2001.

18. Gordon AN, Fleagle JT, Guthrie D, Parkin DE, Gore ME and Lacave AJ: Recurrent epithelial ovarian carcinoma: a randomized phase II study of pegylated liposomal doxorubicin versus topotecan. J Clin Oncol 19: 3312-3322, 2001.

19. Katsumata N, Fujiwara Y, Kamura T, et al: Phase II clinical trial of pegylated liposomal doxorubicin (JNS002) in Japanese patients with Müllerian carcinoma (epithelial ovarian carcinoma, primary carcinoma of fallopian tube, peritoneal carcinoma) having a therapeutic history of platinum-based chemotherapy: A phase II study of the Japanese Gynecologic Oncology Group. Jpn J Clin Oncol 38: 777-785, 2008.

20. Muggia FM, Hainsworth JD, Jeffers S, et al: Phase II study of liposomal doxorubicin in refractory ovarian cancer: antitumor activity and toxicity modification by liposomal encapsulation. J Clin Oncol 15: 987-993, 1997.

21. Gordon AN, Fleagle JT, Guthrie D, Parkin DE, Gore M,Lacave AJ and Mutch D: Interim analysis of a phase III randomized trial of doxil/caelyx (D) versus topotecan (T) in the treatment of patients with relapsed ovarian cancer. Proc Am Soc Clin Oncol: 9: 380, 2000 . 